\title{
Modeling star formation and AGN activity in sub-mJy radio surveys
}

\author{
Claudia Mancuso*; Andrea Lapi, Luigi Danese \\ SISSA-ISAS, Trieste, Italy \\ Gianfranco De Zotti \\ INAF-OAPD, Padova, Italy and SISSA-ISAS, Trieste, Italy
}

\section{Matteo Bonato}

Tufts University, Medford, MA 02155, USA

\begin{abstract}
Although it is widely agreed that the well-known flattening of the radio number counts below $1 \mathrm{mJy}$ is mostly due to star-forming galaxies, a large fraction $(\sim 50 \%)$ of sub-mJy sources are found to host an AGN. The AGN contribution to the observed radio emission is however still debated. While several studies suggest that the dominant contribution comes from star formation in the host galaxy, others suggest that this radio emission is predominantly caused by accretion activity. We have addressed this issue exploiting the model, developed by our team, that deals in a self consistent way with the co-evolution of the star-formation and of the AGN activity. The model allows us to compute the distribution of bolometric luminosities of AGNs hosted by a galaxy with a given star formation rate at a given redshift. Using in addition an updated version of the evolutionary model by Massardi et al. (2010) for radio loud AGNs, we have computed the contribution to the sub-mJy counts at $1.4 \mathrm{GHz}$ by: i) star-forming galaxies without evidence of nuclear activity; ii) star-forming galaxies hosting an AGN, assuming that the radio emission comes from star-formation; iii) radio loud AGNs. We have also computed the redshift and luminosity distributions of population i) and ii) at the detection limit of the Extended Chandra Deep Field South VLA survey. We find that the data do not require a large 'radio-quiet' AGN contribution to the radio emission, although a sub-dominant contribution, compared to that due to star formation, cannot be ruled out. We also investigated the possibility that the relationship between radio emission and star formation rate may be slightly non-linear.
\end{abstract}

EXTRA-RADSUR2015 (*)

20-23 October 2015

Bologna, Italy

(*) This conference has been organized with the support of the Ministry of Foreign Affairs and International Cooperation, Directorate General for the Country Promotion (Bilateral Grant Agreement ZA14GR02 - Mapping the Universe on the Pathway to SKA)

\footnotetext{
* Speaker.

†E-mail: cmancuso@ sissa.it
} 


\section{Framework}

In Mancuso et al. (2015) we presented number counts and redshift distributions of the various populations of star-forming galaxies at the detection limits foreseen for surveys with the SKA and its precursors. In the case of high- $z$ galaxies we relied on the galaxy-AGN co-evolution model developed at SISSA (Granato et al., 2004; Lapi et al., 2006, 2011, 2014). The model hinges upon the results of intensive $N$-body simulations and semi-analytic studies, takes into account the energy feedback from supernovae and from the active nucleus, and includes a self consistent treatment of the chemical evolution of the ISM, calculated using the standard equations and stellar nucleosynthesis prescriptions. The chemical evolution controls the evolution of the dust abundance, hence the dust absorption and re-emission. We also implemented the effect of gravitational lensing, showing that the highest redshift tails of the distributions at the detection limits of planned deep surveys comprise a substantial fraction of strongly lensed galaxies.

For the low- $z$ galaxies instead we adopted the phenomenological approach described in Cai et al. $(2013,2014)$, that accurately reproduces the evolution of the star formation rate (SFR) function across the cosmic history, as derived from UV, IR and Ly $\alpha$ data, taking into account dust attenuation and re-emission.

The SFR functions were then translated into radio luminosity functions using the relationships between radio luminosity and SFR derived by Murphy et al. (2011, 2012). Having at disposal the radio luminosity functions we addressed the issue of the presence of AGNs in star forming galaxies (SFGs), irrespective of whether or not they contribute significantly to the radio power of their hosts.

This was done in a statistical sense, i.e. considering the probability of finding an AGN with bolometric luminosity $L_{\text {bol }}$ inside a galaxy with given SFR at a given redshift. In the case of high- $z$ galaxies such probability was computed by Bonato et al. (2014) based on the SISSA AGN-galaxy co-evolution model. According to this model, the gas infall to the galactic center, where the black hole resides, is regulated by a gas reservoir, where the gas accumulates before being accreted by the central black hole (BH). For a large fraction of the AGN lifetime the accretion occurs at the Eddington limit. In this phase there is no proportionality between accretion rate and SFR.

For the low- $z$ galaxies instead we rely on empirical estimations of the BH luminosity at a given SFR of the host galaxy. At low redshifts the AGN emission is generally sub-Eddington also by large factors and a correlation between accretion rate and SFR can be expected and is indeed predicted by several models. We have exploited the mean relation between the average black hole accretion rate (BHAR), hence the AGN bolometric luminosity, $L_{\mathrm{bol}}$, and the SFR derived by Chen et al. (2013):

$$
\frac{\mathrm{BHAR}}{M_{\odot} \mathrm{yr}^{-1}}=10^{-(3.72 \pm 0.51)}\left(\frac{\mathrm{SFR}}{M_{\odot} \mathrm{yr}^{-1}}\right)^{1.05 \pm 0.33}
$$

This relation was derived using a sample of 1767 far-IR selected galaxies in a region covered by deep X-ray observations with Chandra, with redshifts in the range $0.25<z<0.8$. The accretion luminosity was derived applying to X-ray data a bolometric correction $L_{\mathrm{bol}} / L_{X}=22.4$ and adopting a mass to light conversion efficiency of 0.1. A better consistency of the model with the data discussed below was achieved decreasing the coefficient by 0.2 dex, well within its uncertainty.

Following Bonato et al. (2014), the probability of finding an AGN with accretion rate BHAR inside a star-forming galaxy with a given SFR was computed assuming a Gaussian distribution 
around the mean BHAR given in Eq. (1.1), decreased by 0.2 dex, with a dispersion $\sigma=0.69$ dex, consistent with the distribution of Chen et al. (2013) data points. As shown by Bonato et al. (2014), under these assumptions we can reproduce the bolometric luminosity functions of AGNs by Delvecchio et al. (2014) at $z \leq 1.5$ (at higher $z$ they are reproduced by the Cai et al. (2013) co-evolution model).

\section{Results}

The aim of this work was to check whether our galaxy-AGN co-evolution model, applied by Mancuso et al. (2015) to derive counts and redshift distributions of star-forming galaxies, can also account for the statistics of radio quiet (RQ) AGNs detected by the Extended Chandra Deep Field-South Very Large Array (VLA) survey at $1.4 \mathrm{GHz}$ (Padovani et al., 2015), assuming that the measured radio emission is entirely due to star-formation in their host galaxies. This assumption was motivated by the fact that Mancuso et al. (2015) were able to accurately reproduce the submJy source counts at several radio frequencies taking into account only radio loud (RL) AGNs and star-forming galaxies, i.e. neglecting the radio emission of RQ AGNs.

The Padovani et al. (2015) sample is a sub-sample of that described by Bonzini et al. (2013). It includes the 765 radio sources, over an area of $0.285 \mathrm{deg}^{2}$, for which enough ancillary multiwavelength data to provide reasonable photometric redshifts are available. This sub-sample was used to derive the number counts. To study the evolution of RL and RQ AGNs, the sample was further cut in order to maximize the fraction of sources with redshift. This was achieved by keeping only sources with flux density at $3.6 \mu \mathrm{m}$ larger than $1 \mu \mathrm{Jy}$. In this way they obtained a sample of 680 radio sources $92 \%$ of which have redshift. For the remaining $8 \%$ the redshift was estimated using an empirical relation between the $3.6 \mu \mathrm{m}$ flux density and the redshift, namely:

$$
\log z=-0.677 \log f_{3.6 \mu m}+1.185
$$

The model differential counts of star-forming galaxies and of RL AGNs were computed as in Mancuso et al. (2015). To evaluate the counts of RQ AGNs associated to star-forming galaxies predicted by the model, we first derived their radio luminosity functions convolving the SFR functions with the probability functions described in Sect. 1, under the assumption that the radio emission is due to star formation. Only AGNs with X-ray luminosity greater than $10^{42} \mathrm{erg} \mathrm{s}^{-1}$, i.e. with a BHAR $>3.9 \times 10^{-3} M_{\odot} \mathrm{yr}^{-1}$ were taken into account: lower X-ray luminosities can be accounted for by star formation. The results, shown in the left hand panel of Fig. 1, are in satisfactory agreement with observational estimates. This supports the assumption that the radio emission of RQ AGNs is sub-dominant compared to that of their host galaxies, although the substantial uncertainties affecting both theoretical and observational estimates do not allow us to set strong constraints.

Padovani et al. (2015) have also worked out estimates of $1.4 \mathrm{GHz}$ luminosity functions of RQ AGNs, assigning to them the entire radio luminosity of the associated sources. The right-hand panel of Fig. 1 compares these estimates with the luminosity functions of star-forming galaxies hosting an AGN with $L_{X}>10^{42} \mathrm{erg} \mathrm{s}^{-1}$, given by the model. The agreement is good at low redshift, while at $z>1-1.5$ the model predictions are somewhat lower that the data points, which however suffer of large uncertainties. 
A similar discrepancy was found by Bonato et al. (in preparation) in their analysis of the redshift dependent luminosity functions of SFGs derived from the deep VLA survey of the COSMOS field (Schinnerer et al., 2007; Bondi et al., 2008). Bonato et al. found that a comparison between the radio luminosity functions of SFGs and their SFR functions indicates a slightly non-linear relation between the SFR and the radio synchrotron luminosity:

$$
\bar{L}_{\mathrm{sync}} \simeq 1.9 \times 10^{28}\left(\frac{\mathrm{SFR}}{\mathrm{M}_{\odot} \mathrm{yr}^{-1}}\right)^{1.2}\left(\frac{v}{\mathrm{GHz}}\right)^{-0.85}\left[1+\left(\frac{v}{20 \mathrm{GHz}}\right)^{0.5}\right]^{-1} \mathrm{erg} \mathrm{s}^{-1} \mathrm{~Hz}^{-1} .
$$

For comparison, the relation used by Mancuso et al. (2015) is:

$$
\bar{L}_{\mathrm{sync}} \simeq 1.9 \times 10^{28}\left(\frac{\mathrm{SFR}}{\mathrm{M}_{\odot} \mathrm{yr}^{-1}}\right)\left(\frac{v}{\mathrm{GHz}}\right)^{-0.85}\left[1+\left(\frac{v}{20 \mathrm{GHz}}\right)^{0.5}\right]^{-1} \mathrm{erg} \mathrm{s}^{-1} \mathrm{~Hz}^{-1} .
$$

We have then repeated the analysis using Eq. (2.2). The results are shown in Fig. 2. The agreement of the model with observational estimates of the luminosity functions improves at high redshifts. On the other hand, the model counts exceed the observational estimates by Padovani et al. (2015), being still consistent with the $P(D)$ estimates by Condon et al. (2012) and Vernstrom et al. (2014).

Figure 3 compares the cumulative redshift distributions for SFGs hosting or not a RQ AGN, in the Padovani et al. (2015) sample, with the distributions yielded by the model adopting either the linear (left-hand panel) or the non-linear (right-hand panel) relationship between $\bar{L}_{\text {sync }}$ and SFR. We obtained the observational estimates for each source population using the catalogue published by Bonzini et al. (2013). As expected, the linear relationship leads to a substantial under-prediction of the distribution for $z \gtrsim 0.3$. The non-linear relationship performs much better up to $z \simeq 1$ but over-predicts the distribution at higher redshifts.
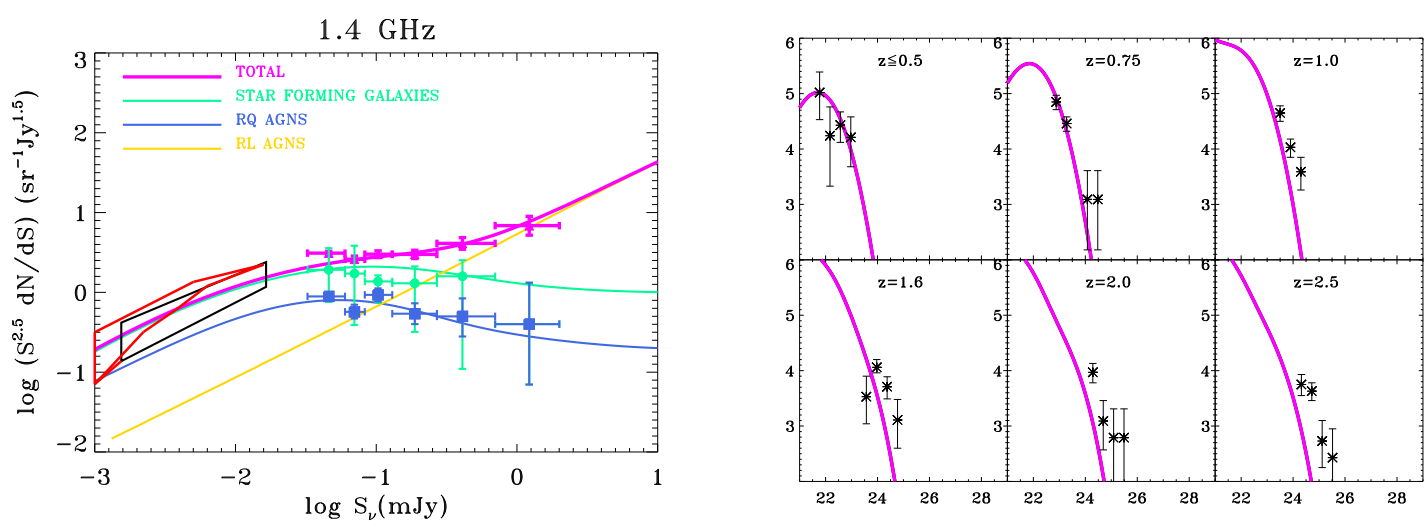

Figure 1: Left panel: Euclidean-normalized number counts at $1.4 \mathrm{GHz}$ of SFGs (green line and data points), of SFGs hosting RQ AGNs (referred to as 'RQ AGNs'; blue line and data points), of RL AGNs (yellow line) and total counts (magenta line and data points). The model counts of SFGs and of RQ AGNs have been computed using the linear proportionality between SFR and radio-synchrotron luminosity [Eq. (2.3)]. The black and red polygons on the left-hand side show the results of the $P(D)$ analyses by Condon et al. (2012) and Vernstrom et al. (2014), respectively. The data points are from Padovani et al. (2015). Right panel: Luminosity functions of SFGs hosting RQ AGNs for different redshift bins. Again Eq. (2.3) was adopted to compute the model luminosity functions. The data points are from Padovani et al. (2015). 

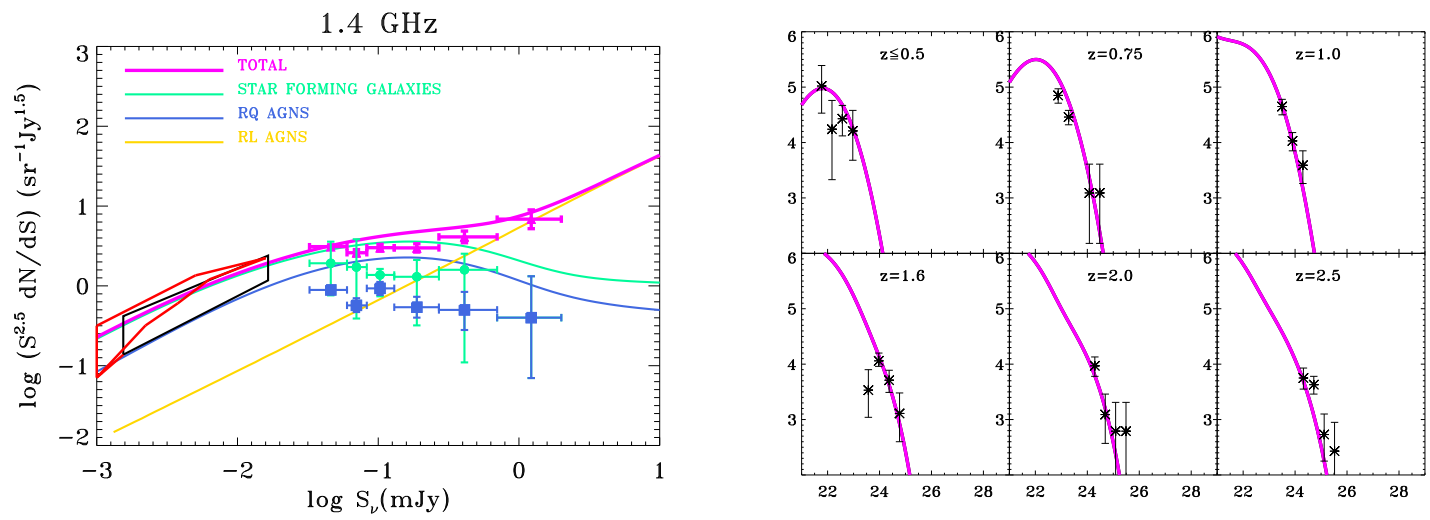

Figure 2: Same as in Fig. 1 but using the non linear relation between synchrotron luminosity and SFR [Eq. (2.2)].
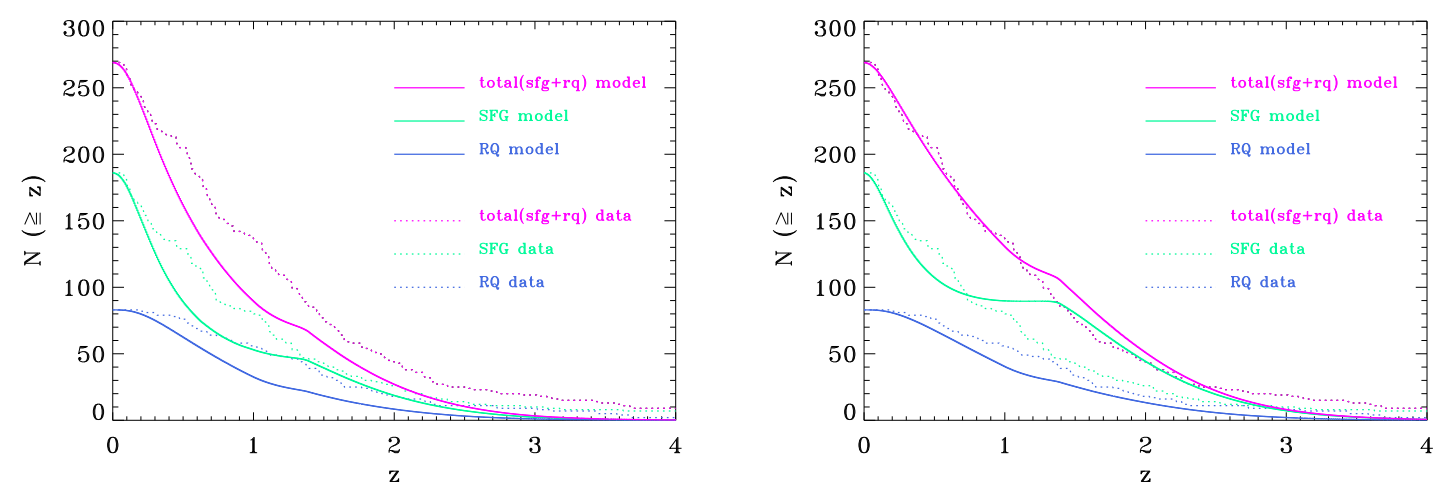

Figure 3: Left panel: Comparison of observational determinations of the cumulative redshift distributions of star-forming galaxies, hosting or not RQ AGNs, with model predictions in the case of linear relationship between $\bar{L}_{\text {sync }}$ and SFR. The observational estimates were obtained using the catalog by Bonzini et al. (2013). Right panel: Same as in the left-hand panel, but for the case of a non-linear proportionality between SFR and radio-synchrotron luminosity. The data are from Bonzini et al. (2013).

\subsection{Source classification}

The classification of sources as RL or RQ AGNs or star-forming galaxies without an active nucleus is a tricky process. Padovani et al. (2015) adopted the classification done by Bonzini et al. (2013) which starts from consideration of the logarithm of the ratio between the observed $24 \mu \mathrm{m}$ and $1.4 \mathrm{GHz}$ flux densities:

$$
q_{24 \mathrm{obs}}=\log _{10} \frac{S_{24 \mu \mathrm{m}}}{S_{1.4 \mathrm{GHz}}} .
$$

The distribution of $q_{24 \text { obs }}$ as a function of $z$ allowed Bonzini et al. (2013) to define an 'SFG locus' based on the radio-far-IR correlation for these objects. The redshift-dependent boundaries of this locus were computed assuming a radio spectral index of $0.7\left(S_{v} \propto v^{-0.7}\right)$ and the Spectral Energy Distribution (SED) of the local starburst galaxy M82. Sources below the lower boundary, i.e. sources with a radio excess, are classified as RL AGNs.

This classification scheme, while well justified, is unavoidably liable to substantial uncertain- 
ties. As illustrated by Fig. 4, the distribution of sources in the $q_{24 \mathrm{obs}}-z$ plane is continuous, so that there is no 'natural' boundary between the loci of SFGs and of RL AGNs. The definition of the boundary depends on the choice of the SED. That of M82 is not necessarily a good choice. As shown e.g. by Smith et al. (2011), the SEDs of SFGs are luminosity-dependent and high- $z$ galaxies in the considered sample have IR luminosities much higher than M82. Figure 4 shows, as an example, how the SFG locus changes if we adopt the SED of the $z=2.3$ galaxy SMM J2135-0102, 'The Cosmic Eyelash' (Ivison et al., 2010) in place of that of M82.

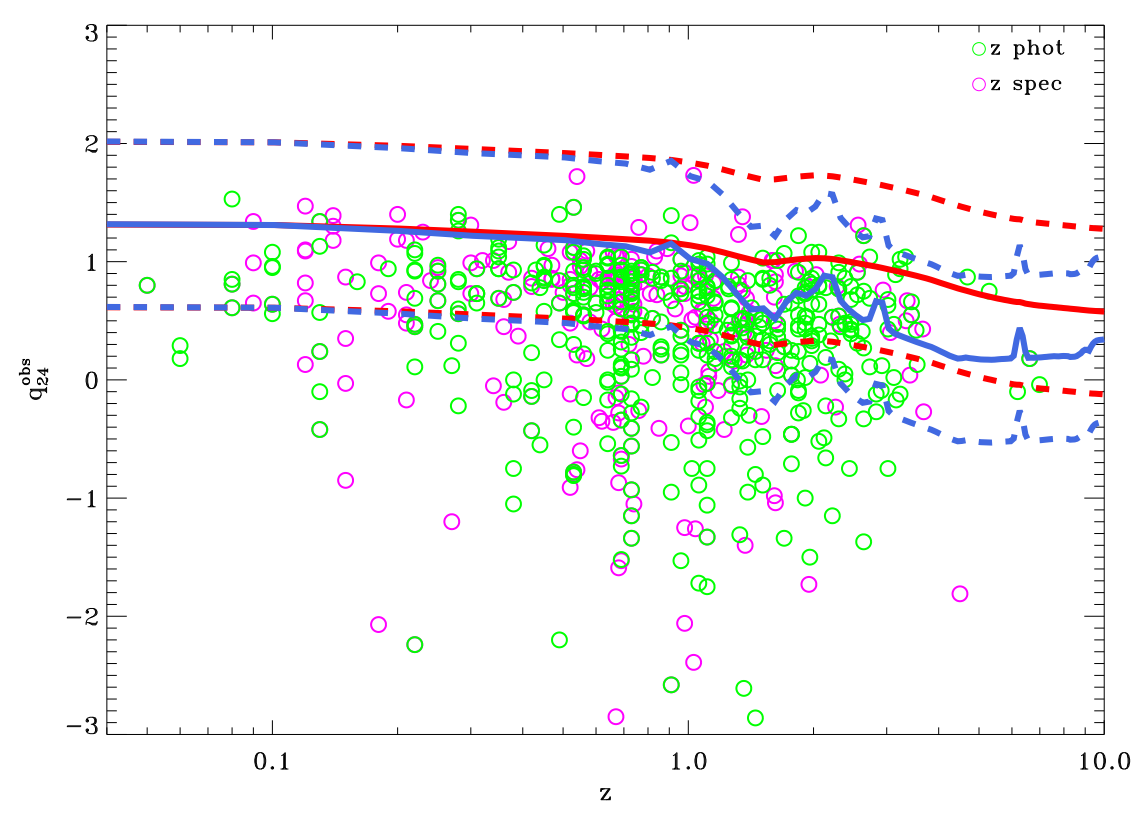

Figure 4: Comparison of the 'SFG loci' based on the $q_{24 \mathrm{obs}}$ index as a function of $z$ for 2 choices of the galaxy SED: that of the nearby, low luminosity starburst galaxy M82, used by (blue lines)Bonzini et al. (2013) and that of the $z=2.3$ galaxy SMM J2135-0102, 'The Cosmic Eyelash' (red lines). The data are from Bonzini et al. (2013)

The selection of RQ AGNs entails an additional complication. X-ray luminosities larger than $10^{42} \mathrm{erg} \mathrm{s}^{-1}$ can be unambiguously attributed to nuclear activity. Thus sources within the SFG locus having such X-ray luminosities do host a RQ AGN. However, only a minority of sources in the Padovani et al. (2015) sample are detected in X-rays. The RQ AGN classification of the other sources within the SFG locus relies on the Spitzer/IRAC color-color diagram, $\log \left(S_{8 \mu \mathrm{m}} / S_{4.5 \mu \mathrm{m}}\right)$ versus $\log \left(S_{5.8 \mu \mathrm{m}} / S_{3.6 \mu \mathrm{m}}\right)$. The selection criterion adopted by Bonzini et al. (2013) is designed to minimize the contamination of the RQ AGN sample from SFGs. However, on one hand some contamination is unavoidable and, on the other hand, the price to be paid choosing a strict selection criterion is incompleteness. As pointed out by Bonzini et al. (2013), their selection method easily misses low luminosity AGNs and is also incomplete for luminous AGNs with heavy obscuration and particularly bright host galaxies.

In view of these uncertainties, detailed comparisons of model predictions with observational determinations of the statistical properties of each source population must be dealt with caution. 


\section{Conclusions}

Exploiting the rich multi-frequency information content on sources detected by the Extended Chandra Deep Field-South VLA survey at $1.4 \mathrm{GHz}$, Padovani et al. (2015) have derived deep counts of different source populations (RL AGNs, SFGs and RQ AGNs) as well as luminosity functions of RQ AGNs at several redshifts. RQ AGNs were defined as sources with radio to infrared luminosity ratios within the range covered by the radio-IR correlation obeyed by SFGs, and having either Xray luminosities or IR colors indicative of nuclear activity. This leaves open the issue of whether the observed radio emission has to be attributed to the RQ AGNs, to the host galaxy or to a combination of both.

Padovani et al. (2015) found that the evolution of RQ AGNs in the radio band is similar to that of SFGs, suggesting that the radio emission is due to host galaxies. We have taken a different approach to investigate the origin of the radio emission of these sources. Our approach exploits the galaxy-AGN co-evolution model successfully tested by Mancuso et al. (2015) against data from multi-frequency radio surveys and used to make predictions for radio source counts down to nJy levels, and for redshift distributions at the detection limits of planned surveys with the SKA and its precursors.

The model predicts the evolution with cosmic time of the SFR function and links it to the redshift-dependent radio luminosity function of SFGs via the relation between radio luminosity and SFR. It also provides the probability that a galaxy of given SFR at given $z$ hosts an AGN, as a function of the AGN bolometric luminosity. We have then used the model to compute the counts of SFGs hosting a RQ AGN assuming that the observed radio emission is entirely due to the host galaxies.

A comparison of model predictions with the counts of SFGs and of RQ AGNs observationally determined by Padovani et al. (2015) shows good agreement, supporting the conclusion that the contribution of RQ AGNs to the observed radio emission must be sub-dominant, although the still relatively large uncertainties do not allow us to set strict limits.

We have also compared the radio luminosity function of RQ AGNs at several redshifts with the estimates by Padovani et al. (2015). The agreement is good at low redshift, while at $z>1-1.5$ the model predictions are somewhat low. The same conclusion is obtained by comparing the predicted with the observed redshift distribution. The discrepancy can be reduced if the relation between the SFR and the radio synchrotron luminosity slightly deviates from linearity. On the other hand, the non linear relation yields source counts exceeding the observational estimates by Padovani et al. (2015).

We end with a note of caution. The source classification allowed by the available data is endowed with substantial uncertainties. Therefore we must be careful not to over-interpret the comparisons between model predictions and observational estimates.

\section{Acknowledgements}

We acknowledge financial support from ASI/INAF Agreement 2014-024-R.0 for the Planck LFI activity of Phase E2, from PRIN INAF 2012, project "Looking into the dust-obscured phase of galaxy formation through cosmic zoom lenses in the Herschel Astrophysical Large Area Survey" 
and from PRIN INAF 2014, project "Probing the AGN/galaxy co-evolution through ultra-deep and ultra-high resolution radio surveys".

\section{References}

Bonato, M., Negrello, M., Cai, Z.-Y., et al. 2014, MNRAS, 438, 2547

Bondi, M., Ciliegi, P., Schinnerer, E., et al. 2008, ApJ, 681, 1129

Bonzini, M., Padovani, P., Mainieri, V., et al. 2013, MNRAS, 436, 3759

Cai, Z.-Y., Lapi, A., Bressan, A., et al. 2014, ApJ, 785, 65

Cai, Z.-Y., Lapi, A., Xia, J.-Q., et al. 2013, ApJ, 768, 21

Chen, C.-T. J., Hickox, R. C., Alberts, S., et al. 2013, ApJ, 773, 3

Condon, J. J., Cotton, W. D., Fomalont, E. B., et al. 2012, ApJ, 758, 23

Delvecchio, I., Gruppioni, C., Pozzi, F., et al. 2014, MNRAS, 439, 2736

Granato, G. L., De Zotti, G., Silva, L., Bressan, A., \& Danese, L. 2004, ApJ, 600, 580

Ivison, R. J., Swinbank, A. M., Swinyard, B., et al. 2010, A\&A, 518, L35

Lapi, A., Shankar, F., Mao, J., et al. 2006, ApJ, 650, 42

Lapi, A., González-Nuevo, J., Fan, L., et al. 2011, ApJ, 742, 24

Lapi, A., Raimundo, S., Aversa, R., et al. 2014, ApJ, 782, 69

Mancuso, C., Lapi, A., Cai, Z.-Y., et al. 2015, ApJ810,72

Massardi, M., Bonaldi, A., Negrello, M., et al. 2010, MNRAS, 404, 532

Murphy, E. J., Condon, J. J., Schinnerer, E., et al. 2011, ApJ, 737, 67

Murphy, E. J., Bremseth, J., Mason, B. S., et al. 2012, ApJ, 761, 97

Padovani, P., Bonzini, M., Kellermann, K. I, et al. 2015, MNRAS,452,1263

Schinnerer, E., Smolčić, V., Carilli, C. L., et al. 2007, ApJ, 172, 46

Smith, D. J. B., Dunne, L., Maddox, S. J., et al. 2011, MNRAS, 416, 857

Vernstrom, T., Scott, D., Wall, J. V., et al. 2014, MNRAS, 440, 2791 